\title{
The duality between the anti-exchange closure operators and the path independent choice operators on a finite set
}

\author{
Bernard Monjardet* Vololonirina Raderanirina *
}

\begin{abstract}
In this paper, we show that the correspondence discovered by Koshevoy ([18]) and Johnson and Dean ([15],[16]) between anti-exchange closure operators and path independent choice operators is a duality between two semilattices of such operators. Then we use this duality to obtain results concerning the "ordinal" representations of path independent choice functions from the theory of anti-exchange closure operators.
\end{abstract}

Key-words: Anti-exchange closure operator, choice function, convex geometry, path independence, partial order, semilattice.

${ }^{*}$ CERMSEM, Université Paris 1, 106-112 boulevard de l'Hopital, 75647 Paris Cedex 13, France. Email : (monjarde, raderani)@univ-paris1.fr 


\section{Introduction}

Let $S$ be a finite ${ }^{1}$ set. A closure operator $k$ on $S$ is an idempotent ${ }^{2}$ and isotone ${ }^{3}$ map associating to any subset $A$ of $S$ a subset $k(A)$ containing A. A choice operator $c$ on $S$ is a map associating to any subset $A$ of $S$ a subset $c(A)$ contained in $A$. Closure operators abound in mathematics, for instance because any Moore family on $S$ - i.e. any family $\mathcal{F}$ of subsets of $S$ containing $S$ and the intersection of any two members of the family - defines a closure operator on $S$ (conversely the family of closed sets of a closure operator $k$ - i.e. the subsets $K$ of $S$ satisfying $k(K)=K$ - is a Moore family). Moreover the theory of closure operators is closely linked to the theory of lattices : the family of the closed sets of a closure operator is a (complete) lattice with respect to the inclusion order between subsets and, conversely, any (complete) lattice can be represented as the lattice of the closed sets of a closure operator. Choice operators appear in several fields of mathematics, but they have been especially studied in the theory of the revealed preference in microeconomics where, under the name of choice functions, they model the choice behavior of a consumer (for surveys on this theory see [30], [25], or [1]).

A first link between closure operators and choice operators appears in Malishevski's papers ([21], [22]), where he compares some representation properties of closure operators and of the choice operators satisfying the so-called path independence property. This property, introduced by Plott ([26]), says that $c(A \cup B)=c(c(A) \cup c(B))$ for all $A, B$ subsets of $S$. Yet a fundamental connection between the theory of closure operators and the theory of choice operators was found by Koshevoy ([18]) who proved the existence of a natural correspondence between the so-called anti-exchange closure operators and the path independent choice operators. This correspondence was also discovered with a different formulation by Johnson and Dean $([15],[16])$. The first aim of this paper is to strengthen this correspondence by showing that it induces a duality between the semilattice of the anti-exchange closure operators and the semilattice of the path independent choice operators. The second aim is to show how this duality allows us to obtain immediately results in the theory of path independent choice operators from classical or non-classical results in the theory of anti-exchange closure operators. For

\footnotetext{
${ }^{1}$ The notion of closure and choice operators defined below can be defined on arbitrary sets, but in all this paper we consider only finite sets.

${ }^{2} k^{2}=k$.

${ }^{3} A \subseteq B$ implies $k(A) \subseteq k(B)$.
} 
instance in this way we obtain the Aizerman and Malishevski ([3]) representation result of path independent choice operators by linear orders.

The paper is organized as follows. In section 2 we recall some definitions and results on operators, closure operators and especially anti-exchange closure operators, choice operators and especially path independent choice operators, and we give some preliminary results. In particular we prove some general facts about a significant tool introduced by Koshevoy ([18]) and called here the Koshevoy map, and we give several new characterizations of anti-exchange closure operators linked to the notion of extreme elements of a subset (with respect to an operator). In section 3 we first present the meet-semilattice of anti-exchange closure operators and the join-semilattice of path independent choice operators, then we prove that these two semilattices are dual. Section 4 uses this duality to obtain the results on the "ordinal" representations of path independent closure operators. We conclude by putting these results in the framework of the theory of choice functions and by mentioning some research topics.

Finally, since the theory of anti-exchange closure operators plays an important role in this paper it is worthwhile to mention that it is the same as the theory of convex geometries (a convex geometry is the family of closed sets of an anti-exchange closure operator) and that it is closely related to the theory of the so-called lower locally (or meet) distributive lattices : a convex geometry is such a lattice and any such lattice is isomorphic with a convex geometry. Surveys on this theory can be found in [13], [11] and [24], and uses of this theory in other domains of social sciences can be found in [12], [17] and [5].

\section{Closure and choice operators}

This section contains definitions, recalls and preliminary results. The first subsection gives the definitions of several properties of operators, and some relations between these properties, especially those concerning the "Koshevoy map". The two other subsections consider successively the closure operators and especially the anti-exchange closure operators, and the choice operators and especially the path independent choice operators.

N.B. The symbol + denotes the disjoint union of sets. For $A \subseteq B$ subsets of a set $S,[A, B]$ denotes the set $\{X \subseteq S: A \subseteq X \subseteq B\}$. 


\section{$2.1 \quad$ Operators}

Definition 1 Let $S$ be a finite set. An operator $f$ on $S$ is a map from $2^{S}$ into $2^{S}$ (where $2^{S}$ denotes the set of all subsets of $S$ ).

We denote by $\mathcal{F}_{f}$ the set $\{f(A), A \subseteq S\}$ of all the images of the elements of $2^{S}$ by the operator $f$. For $A \subseteq S$, we denote by $f^{-1}[f A]$ the set $\{X \subseteq S: f(X)=f(A)\}$ of all inverse images of $f(A)$.

The composition of two operators $f$ and $g$ on $S$ is written $f g$, where $f g(A)=$ $f(g(A))$. If $g=f, f g$ is written $f^{2}$.

Definition 2 Let $f$ be an operator on $S$.

(1) $f$ is extensive if $A \subseteq f(A), \forall A \subseteq S$.

(2) $f$ is contracting (or shrinking) if $f(A) \subseteq A, \forall A \subseteq S$.

(3) $f$ is isotone if $A \subseteq B$ implies $f(A) \subseteq f(B), \forall A, B \subseteq S$.

(4) $f$ is idempotent if $f^{2}(A)=f(A), \forall A \subseteq S$.

(5) $f$ is convex if $f^{-1}[f A]$ is convex for every $A \subseteq S$, i.e. if $[f(A)=f(B)$ and $A \subseteq X \subseteq B]$ imply $f(A)=f(X)$.

(7) $f$ satisfies the property (MAX) if $f^{-1}[f A]$ has a maximum element for every $A \subseteq S$, i.e. if there exists $M \subseteq S$ with $f(M)=f(A)$, and $f(X)=f(A)$ imply $X \subseteq M$.

(8) $f$ satisfies the outcast property (O) if $A \cap f(A) \subseteq B \subseteq A \cup f(A)$ implies $f(B)=f(A), \forall A, B \subseteq S$.

(9) $f$ satisfies the heritage property (H) if $f(B) \cap A \subseteq f(A), \forall A \subseteq B \subseteq$ $S$.

(10) $f$ satisfies the path independence property (PI), if $f(A \cup B)=f(f(A) \cup f(B)), \forall A, B \subseteq S$.

Remark 1 We use the term outcast property for the property $(\mathrm{O})$ since when $f$ is contracting it becomes the classical outcast property of the theory of choice functions : $f(A) \subseteq B \subseteq A$ implies $f(A)=f(B), \forall A, B \subseteq S$. Such a property has been also called the Aizerman property $([25])$ and the Nash axiom [30]. The term heritage comes also from the theory of choice functions where one uses alternative terms like Chernoff or property $\alpha$ [28].

We define a map between operators, introduced by Koshevoy ([18]) and denoted here by $\kappa$.

Definition 3 Let $f$ be an operator on $S . \kappa(f)$, denoted by $\kappa_{f}$, is the operator on $S$ defined by : for every $A \subseteq S$,

$$
\kappa_{f}(A)=\bigcup\{X \subseteq S: f(X)=f(A)\}=\bigcup f^{-1}[f A] .
$$


Thus the Koshevoy map transforms an arbitrary operator into an extensive operator.

Some relations between the above properties of operators can be formulated with the Koshevoy map. These relations and some others are stated in the following proposition.

Proposition 1 Let $f$ be an operator on $S$.

(1) If $f$ is path independent, $f$ is idempotent. If $f$ is idempotent, it satisfies $\kappa_{f} f=\kappa_{f}$.

(2) $f$ satisfies the property $M A X$ (i.e. $f^{-1}[f A]$ has a maximum element for every $A \subseteq S)$ if and only if $f \kappa_{f}=f$, and then $\operatorname{Max}\left(f^{-1}[f A]\right)=\kappa_{f}(A)$ for every $A \subseteq S$.

(3) If $f$ is a contracting operator, then the following holds:

(a) $f$ is idempotent if and only if $f(A)=\operatorname{Min}\left(f^{-1}[f A]\right)$ for every $A \subseteq S$, and if $f$ satisfies the heritage property it is idempotent,

(b) $f$ is idempotent and convex if and only if $f$ satisfies the outcast property,

(c) $f$ satisfies the outcast property and the property (MAX) if and only if $f^{-1}[f A]=\left[f(A), \kappa_{f}(A)\right]$,

(d) $f$ is path independent if and only if $f\left(\cup_{i \in I} A_{i}\right)=f\left(\cup_{i \in I} f\left(A_{i}\right)\right)$ for every family $\left\{A_{i}\right\}_{i \in I}$ of subsets of $S$, if and only if $f(A \cup B)=f(f(A) \cup B)$ for all $A, B \subseteq S$, and if and only if $f$ satisfies the heritage and outcast properties.

Proof (1) The first assertion is obtained by taking $A=B$ in the definition of a path independent operator and the second assertion is an obvious consequence of the definition of $\kappa_{f}$.

(2) Let $A \subseteq S$. If there exists $M \subseteq S$ such that $f(M)=f(A)$ and $f(X)=$ $f(A)$ implies $X \subseteq M$, one has $\kappa_{f}(A)=M$, and $f \kappa_{f}(A)=f(M)=f(A)$. Conversely if $M=\kappa_{f}(A)$ and $f \kappa_{f}(A)=f(M)=f(A)$, then $f(X)=f(A)$ implies $X \subseteq M$, and $M$ is the maximum element of $f^{-1}[f A]$.

(3) (a) If $f$ is contracting and idempotent, $f(A) \in f^{-1}[f A]$ and $f(X)=f(A)$ implies $f(A) \subseteq X$. The converse implication is clear, as well as the second assertion (apply (H) to $c(A) \subseteq A$ ).

(b) Let $f$ be a contracting, idempotent and convex operator. If $f(A) \cap A=$ $f(A)=f^{2}(A) \subseteq B \subseteq A=f(A) \cup A$, then $f(B)=f(A)$ (by convexity). If $f$ satisfies property $(\mathrm{O}), A \cap f(A)=f(A) \subseteq f(A) \subseteq A \cup f(A)=A$ implies $f^{2}(A)=f(A)$, and $A \subseteq X \subseteq B$ with $f(A)=f(B)$ imply $f(B) \cap B=$ $f(B) \subseteq X \subseteq B=f(B) \cup B$ and so $f(X)=f(B)$.

(c) If $f$ satisfies the properties (O) and (MAX), $f^{-1}[f A]$ has a minimum element (by (3a) and (3b)), a maximum element $\kappa_{f}(A)$ (by $(2)$ ) and since 
$f^{-1}[f A]$ is convex (by $\left.(3 \mathrm{~b})\right), f^{-1}[f A]$ is equal to the interval $\left[f(A), \kappa_{f}(A)\right]$. The converse implication is obvious.

(d) These equivalent formulations of the path independence property are classical (and easy to obtain). The last one is due to Aizerman and Malishevski ([3], see also [21]).

A significant property of the Koshevoy operator is the fact that it conserves the path independence property.

Theorem 1 If $f$ is a path independent operator, $\kappa_{f}$ is a path independent operator satisfying $f \kappa_{f}=f$.

Proof We must show that $\kappa_{f}(A \cup B)=\kappa_{f}\left(\kappa_{f}(A) \cup \kappa_{f}(B)\right)$.

We set $f^{-1}[f A]=\left\{X_{1}, \ldots, X_{p}\right\}$ and $f^{-1}[f B]=\left\{Y_{1}, \ldots, Y_{q}\right\}$. So $\kappa_{f}(A) \cup$ $\kappa_{f}(B)=X_{1} \cup \ldots \cup X_{p} \cup Y_{1} \cup \ldots \cup Y_{q}$ and $\kappa_{f}\left(\kappa_{f}(A) \cup \kappa_{f}(B)\right)=\kappa_{f}\left(X_{1} \cup \ldots \cup Y_{q}\right)=$ $\bigcup\left\{Z: f(Z)=f\left(X_{1} \cup \ldots \cup Y_{q}\right)\right\}$. Since $f$ is a path independent operator, this is equal to $\bigcup\left\{Z: f(Z)=f\left(f\left(X_{1}\right) \cup \ldots \cup f\left(Y_{q}\right)\right)\right\}=\bigcup\{Z$ : $f(Z)=f(f(A) \cup f(B))\}=\bigcup\{Z: f(Z)=f(A \cup B)\}=\kappa_{f}(A \cup B)$.

With the above notation, $f \kappa_{f}(A)=f\left(X_{1} \cup \ldots \cup X_{p}\right)=f^{2}(A)=f A$, (indeed $f$ is path independent so it is idempotent).

Remark 2 The fact that $\kappa_{f}$ is a path independent operator does not imply that $f$ is a path independent operator.

Example : Let $S=\{1,2,3\}$. Let $f$ be the operator defined on $S$ by : $f(12)=f(13)=f(1)=1, f(23)=f(2)=2, f(3)=3$ and $f(123)=23$. One has $\mathcal{F}_{\kappa_{f}}=\{\emptyset, 3,23,123\}$. One can check that $\kappa_{f}$ is path independent and that $f$ is not (take $A=12$ and $B=13$ ).

\subsection{Closure operators}

Definition 4 Let $S$ be a finite set. A closure operator $k$ on $S$ is an idempotent, extensive and isotone operator on $S$.

Let $k$ be a closure operator. A subset $K$ of $S$ is called a closed set (with respect to $k$ ) if $K=k(K)$. Remark that the family $\mathcal{F}_{k}$ of the closed sets of $k$ contains $S$ and is stable by intersection. Such a family is called a Moore family (or a closure system) on $S$.

The following result appears in [29]. We give a proof for completeness. 
Proposition 2 Let $k$ be an extensive operator on $S . k$ is a closure operator on $S$ if and only if $k$ satisfies the path independence property.

Proof Let $k$ be an extensive operator on $S$ and $A, B \subseteq S$.

Assume that $k$ is a closure operator. Since $A, B \subseteq A \cup B$, we have $k(A) \cup k(B) \subseteq k(A \cup B)$. Then $k(k(A) \cup k(B)) \subseteq k(A \cup B) \subseteq k(k(A) \cup k(B))$ (since $A \subseteq k(A)$ and $B \subseteq k(B))$. So, $k(A \cup B)=k(k(A) \cup k(B)$ ).

Conversely, if $k$ is path independent, then $A \subseteq B$ implies $k(B)=k(A \cup B)=$ $k(k(A) \cup k(B)) \supseteq k(A) \cup k(B) \supseteq k(A)$, and so $k$ is isotone. Moreover, $k(k(A))=k(k(A) \cup k(A))=k(A \cup A)=k(A)$, i.e. $k$ is idempotent. Hence, $k$ is a closure operator.

Since $\kappa_{k}$ is an extensive operator, Theorem 1, Proposition 1 (1) and Proposition 2 give immediately the following result :

Proposition 3 Let $k$ be an operator on $S$. If $k$ satisfies the path independence property, then $\kappa_{k}$ is a closure operator on $S$ satisfying $k \kappa_{k}=k$ and $\kappa_{k} k=\kappa_{k}$.

We now define the anti-exchange closure operators.

Definition 5 Let $k$ be a closure operator on $S . k$ is said to be antiexchange if $k(\emptyset)=\emptyset$ and for every $A \subseteq S, x, y \in S, x, y \notin k(A)$, $y \in k(A+x)$ implies $x \notin k(A+y)$.

There are many characterizations of anti-exchange closure operators, for instance by means of the properties of their families of closed sets, the socalled convex geometries. Before giving the old and new characterizations that we shall use, we need to define the following map $\epsilon$ between operators.

Definition 6 Let $f$ be an operator on $S . \epsilon(f)$, denoted by $\epsilon_{f}$, is the operator defined on $S$ by : for every $A \subseteq S, \epsilon_{f}(A)=\{x \in A: x \notin f(A-x)\}$.

Thus the map $\epsilon$ transforms an arbitrary operator into a contracting operator. When $k$ is a closure operator the set $\epsilon_{k}(A) \subseteq A$ is usually called the set of extreme elements of $A$. In this case one easily sees that:

- $\epsilon_{k}(A)=\{x \in A: k(A-x) \subset k(A)\}$.

- $\epsilon_{k}(K)=\left\{x \in K: K-x \in \mathcal{F}_{k}\right\}$, if $K$ is a closed set of $k$. 
Also, obviously, $\epsilon_{k}^{2}=\epsilon_{k}, k \epsilon_{k}(A) \subseteq k(A)$ and one easily checks that $\epsilon_{k} k(A) \subseteq$ $\epsilon_{k}(A)$. Generally the families $k^{-1}[k A]$ and $\epsilon_{k}^{-1}\left[\epsilon_{k} A\right]$ are incomparable with the family $\left[\epsilon_{k}(A), k(A)\right]$. The following result shows that anti-exchange closure operators are characterized by equalities between these different sets or families.

Theorem 2 Let $k$ be a closure operator on $S$ satisfying $k(\emptyset)=\emptyset$. The following properties are equivalent :

(1) $k$ is an anti-exchange closure operator,

(2) $k=k \epsilon_{k}$ (i.e. the closure of a subset equals the closure of its extreme elements),

(3) $k=k \epsilon_{k} k$ (i.e. a closed set is the closure of its extreme elements),

(4) $\epsilon_{k}=\epsilon_{k} k$ (i.e. the extreme elements of a subset equals the extreme elements of its closure),

(5) the restriction of the operator $\epsilon_{k}$ to the family $\mathcal{F}_{k}$ of closed sets of $k$ is a one-to-one correspondence between $\mathcal{F}_{k}$ and $\epsilon_{k}\left(\mathcal{F}_{k}\right)$ (the family of the sets of the extreme elements of all the closed sets of $k)$,

(6) $\forall A \subseteq S, k^{-1}[k A]=\left[\epsilon_{k}(A), k(A)\right]$,

(7) $\forall A \subseteq S, \epsilon_{k}^{-1}\left[\epsilon_{k} A\right]=\left[\epsilon_{k}(A), k(A)\right]$.

Proof We first prove the equivalence of properties (1), (2), (3), (4) and (5), and then the two equivalences $(1) \Leftrightarrow(6)$ and $(1) \Leftrightarrow(7)$.

$(1) \Rightarrow(2)$ : Let $k$ be an anti-exchange closure operator and assume that (2) is false, i.e. there exists $A \subseteq S$ with $k \epsilon_{k}(A) \subset k(A)$. Then $A \nsubseteq k \epsilon_{k}(A)$ (if not $k \epsilon_{k}(A)=k(A)$ ). So there exists $x \in A-k \epsilon_{k}(A)$. Then $x \notin \epsilon_{k}(A)$ what means $k(A-x)=k(A)$. If $x$ is the unique element of $A$ not in $k \epsilon_{k}(A)$, one has $A-x \subseteq k \epsilon_{k}(A)$, then $k \epsilon_{k}(A)=k(A)$, a contradiction. So, there exists $y \in A, y \neq x$ with $y \notin k \epsilon_{k}(A)$, and then $k(A-y)=k(A)$. Taking then $B=A-\{x, y\}$, one gets $x \in k(B+y)=k(A)$, and $y \in k(B+x)=k(A)$, a contradiction with the fact that $k$ is an anti-exchange closure operator.

$(2) \Rightarrow(3)$ : Obvious (since $k$ is idempotent).

$(3) \Rightarrow(4)$ : Assume that (4) is false, i.e., that there exists $A \subseteq S$ such that $\epsilon_{k} k(A) \subset \epsilon_{k}(A) \subseteq A$. Let $x \in \epsilon_{k}(A)-\epsilon_{k} k(A)$. So, $\epsilon_{k} k(A) \subseteq A-x$, and $k \epsilon_{k} k(A) \subseteq k(A-x) \subset k(A)$. But (3) implies $k \epsilon_{k} k(A)=k(A)$, a contradiction.

$(4) \Rightarrow(3)$ : Assume that (3) is false. Then there exists a closed set $K$ of $k$ such that $k \epsilon_{k}(K) \subset K$. So $\epsilon_{k}(K)$ is strictly contained in a basis of $K$, (i.e. a minimal subset $B$ such that $k(B)=K$ ). By definition of a basis, 
$\epsilon_{k}(B)=B$. So $\epsilon_{k}(K)=\epsilon_{k}(k B) \subset \epsilon_{k}(B)=B$, a contradiction with (4). $(3) \Rightarrow(5)$ : The restriction is surjective by definition.

Assume that there exist $K, K^{\prime} \in \mathcal{F}_{k}$ with $\epsilon_{k}(K)=\epsilon_{k}\left(K^{\prime}\right)$. Then by (3) $k \epsilon_{k}(K)=K=k \epsilon_{k}\left(K^{\prime}\right)=K^{\prime}$.

$(5) \Rightarrow(1)$ : First remark that (since $\epsilon_{k}(\emptyset)=k(\emptyset)=\emptyset$ and $\epsilon_{k}(k A) \subseteq \epsilon_{k}(A)$ )

(5) implies $\epsilon_{k}(A) \neq \emptyset$, for any $A \neq \emptyset$. Assume that $k$ is not an anti-exchange closure operator. Then there exists $A \subseteq S, x, y \notin k(A)=K_{0}$ such that $k(A+x)=k(A+y)=K$. We claim that there exists a closed set $K^{\prime}$ (different from $K$ ) such that $\epsilon_{k}\left(K^{\prime}\right)=\epsilon_{k}(K)$, and then we obtain a contradiction. Indeed, if $z \in K-K_{0}, x \in K-z$ or $y \in K-z$, so $k(K-z)=K$ and $z \notin \epsilon_{k}(K)$. So $t \in \epsilon_{k}(K)$ implies $t \in K_{0}$. But $t \in \epsilon_{k}(K)$ means $K-t \in \mathcal{F}_{k}$, and $(K-t) \cap K_{0}=K_{0}-t \in \mathcal{F}_{k}$, so $t \in \epsilon_{k}\left(K_{0}\right)$ and $\epsilon_{k}(K) \subseteq \epsilon_{k}\left(K_{0}\right)$. If $\epsilon_{k}(K)=\epsilon_{k}\left(K_{0}\right)$ we are done. If not we set $E_{0}=\epsilon_{k}\left(K_{0}\right)-\epsilon_{k}(K)$, and $K_{1}=K_{0}-E_{0}=\bigcap_{t \in E_{0}}\left(K_{0}-t\right) \in \mathcal{F}_{k}$ (since $K_{0}-t \in \mathcal{F}_{k}$ for $t \in E_{0}$ ). By definition, $K_{1}$ contains $\epsilon_{k}(K)$. Also $\epsilon_{k}(K) \subseteq \epsilon_{k}\left(K_{1}\right)$. Indeed $t \in \epsilon_{k}(K)$ implies $K-t \in \mathcal{F}_{k}$ and $(K-t) \cap K_{1}=K_{1}-t \in \mathcal{F}_{k}$. Continuing this procedure we obtain a decreasing chain of closed sets of $k, K_{0} \supset K_{1} \supset \ldots \supset K_{i} \ldots$ such that $\emptyset \subset \epsilon_{k}(K) \subseteq \epsilon_{k}\left(K_{i}\right) \subseteq K_{i}$ for every $i$. Then by finiteness there exists $K_{i}$ such that $\epsilon_{k}\left(K_{i}\right)=\epsilon_{k}(K)$.

We have shown the equivalence between $(1),(2),(3),(4)$ and (5). We now prove the equivalence between $(1),(6)$ and $(7)$

$(1) \Leftrightarrow(6)$ : It is obvious that (6) implies (2). Conversely assume that $\epsilon_{k}(A) \subseteq X \subseteq k(A)$. Then by (2), one gets $k(X)=k(A)$. Now assume that $X \subseteq k(X)=k(A)$. By (4), one gets $\epsilon_{k}(X)=\epsilon_{k} k(X)=\epsilon_{k} k(A)=\epsilon_{k}(A)$, so $\epsilon_{k}(A) \subseteq X$.

$(1) \Leftrightarrow(7):(7)$ implies (4) is obvious. Conversely assume that $\epsilon_{k}(A) \subseteq$ $X \subseteq k(A)$. Then by (2), $k(X)=k(A), \epsilon_{k} k(X)=\epsilon_{k} k(A)$ and by (4), $\epsilon_{k}(X)=\epsilon_{k}(A)$. Now assume that $\epsilon_{k}(A)=\epsilon_{k}(X)$. Then $\epsilon_{k}(A) \subseteq X$, and by $(2), k(A)=k \epsilon_{k}(A)=k \epsilon_{k}(X)=k(X)$, i.e. $X \subseteq k(A)$.

Remark 3 1) The above condition (3) is a classical characterization of an anti-exchange closure operator (see [13]).

2) According to our conventions $\mathcal{F}_{\epsilon_{k}}$ denotes the family $\left\{\epsilon_{k}(A), A \subseteq S\right\}$ of all the images of elements of $2^{S}$ by the operator $\epsilon_{k}$. If $k$ is an anti-exchange closure operator, Theorem $2(4)$ says that $\mathcal{F}_{\epsilon_{k}}$ is equal to the family $\epsilon_{k}\left(\mathcal{F}_{k}\right)$ of the sets of the extreme elements of all the closed sets of $k$. Moreover Theorem $2(5)$ says that $\mathcal{F}_{k}$ and $\mathcal{F}_{\epsilon_{k}}$ are in a one-to-one correspondence. On the other hand, as already said in the introduction, the set $\mathcal{F}_{k}$ of closed sets of an anti-exchange closure operator $k$ is called a convex geometry, and 
the poset $\left(\mathcal{F}_{k}, \subseteq\right)$ is a lower locally distributive lattice. So using the one-toone correspondence between $\mathcal{F}_{k}$ and $\mathcal{F}_{\epsilon_{k}}$, one can transfer the lower locally distributive latticial structure of $\left(\mathcal{F}_{k}, \subseteq\right)$ to $\mathcal{F}_{\epsilon_{k}}$. To do that it suffices to define an order on $\mathcal{F}_{\epsilon_{k}}$ by : $\epsilon_{k}(K) \leq \epsilon_{k}\left(K^{\prime}\right)$ if and only if $K \subseteq K^{\prime}$. Then $\left(\mathcal{F}_{\epsilon_{k}}, \leq\right)$ is also a lower locally distributive lattice isomorphic with $\left(\mathcal{F}_{k}, \subseteq\right)$. This point of view is developed by Johnson and Dean ([15], [16]).

3) For more details on closure operators and their lattices, see [8].

\subsection{Choice operators}

Definition 7 Let $S$ be a finite set. A choice operator $c$ on $S$ is a contracting operator on $S$ satisfying $c(A) \neq \emptyset$ for every $A \neq \emptyset$.

If $c$ is a choice operator on $S$, we denote by $\mathcal{F}_{c}$ the set of all chosen sets on $S$.

Example : Let $k$ be a closure operator on $S$. If for every non-empty subset $A$ of $S$, the set $\epsilon_{k}(A)$ of extreme elements of $k(A)$ is non-empty, $\epsilon_{k}$ is a choice operator. The following result gives characterizations of such a closure operator.

Proposition 4 Let $k$ be a closure operator on $S$. The following conditions are equivalent :

(1) $\epsilon_{k}$ is a choice operator (i.e. $\epsilon_{k}(A)$ is non-empty for A non-empty),

(2) for any closed set $K$ of $k$, there exists $x \in K$ such that $K-x$ is a closed set of $k$,

(3) there exists a chain $\emptyset=K_{0} \subset K_{1} \subset \ldots K_{i} \subset \ldots \subset S$ of closed sets of $k$ such that $\left|K_{i+1}\right|=\left|K_{i}\right|+1$, for $i=0, \ldots,|S|$.

Proof $(1) \Rightarrow(2)$ : By $(1) \epsilon_{k}(K)$ is non-empty for every non-empty closed set $K$ of $k$ and, if $x \in \epsilon_{k}(K), K-x$ is a closed set of $k$.

$(2) \Rightarrow(3)$ : Obvious (begin with $S$ ).

$(3) \Rightarrow(1)$ : Since $\epsilon_{k} k(A) \subseteq \epsilon_{k}(A)$ for every $A \subseteq S$, it suffices to prove that $\epsilon_{k}(K)$ is non-empty for every closed set $K$ of $k$. Let $K$ be a closed set of $k$, and $K^{\prime}$ the minimal closed set of the chain such that $K \subseteq K^{\prime}$, and $x \in K^{\prime}$ such that $K^{\prime}-x$ belongs to the chain. Then, $K \nsubseteq K^{\prime}-x, x \in K$ and $K \cap K^{\prime}-x=K-x$ is a closed set of $k$. Thus $x$ belongs to $\epsilon_{k}(K)$.

Remark 4 A family of subsets of $S$ satisfying the above condition (2) is called an accessible system in [19]. 
An interesting fact is that the choice operator $\epsilon_{k}$ associated with a closure operator $k$ satisfies the heritage property. Indeed, this property is true even if $\epsilon_{k}$ is not a choice operator.

Proposition 5 Let $k$ be a closure operator on $S$. The associated contracting operator $\epsilon_{k}$ satisfies the heritage property.

Proof Let $A \subseteq B \subseteq S$. We show that $\epsilon_{k}(B) \cap A \subseteq \epsilon_{k}(A)$. Assume that $x \in \epsilon_{k}(B) \cap A$, i.e. $x \notin k(B-x)$ and $x \in A$. Then, $A-x \subseteq B-x$, $x \notin k(A-x)$ and so $x \in \epsilon_{k}(A)$.

Remark 5 The classical result recalled in Proposition 1 (3d) says that a choice operator is path independent if and only if it satisfies the heritage $(\mathrm{H})$ and outcast $(\mathrm{O})$ properties. Another interesting characterization of path independence has been given by Johnson and Dean ([15]) : a choice operator $c$ is path independent if and only if it satisfies the heritage property and the property $c^{-1}[c(A)]=\left[c(A), \kappa_{c}(A)\right]$ for every $A \subseteq S$ (called the quotient property $(Q P)$ in $[15])$. Indeed by Proposition $1(3 \mathrm{c}),(\mathrm{QP}) \Leftrightarrow[(\mathrm{O})+$ $(\mathrm{MAX})]$. So $[(\mathrm{H})+(\mathrm{QP})] \Leftrightarrow[(\mathrm{H})+(\mathrm{O})+(\mathrm{MAX})] \Leftrightarrow[(\mathrm{PI})+(\mathrm{MAX})] \Leftrightarrow$ (PI) (Theorem 1 and Proposition 1 (2)).

\section{The duality between the anti-exchange closure operators and the path independent choice op- erators}

In this section we first present the semilattice structure of the sets of all anti-exchange closure operators and of all path independent choice operators. We then show that these two semilattices are dual.

Recall that a meet-semilattice (respectively join-semilattice) $L$ is a poset $(L, \leq)$ such that for all $x, y$ in $L$, the meet (respectively the join) of $x$ and $y$ exists, i.e. such that $x$ and $y$ have a greatest lower bound (respectively least upper bound) denoted by $x \wedge y$ (respectively $x \vee y$ ). In a (finite) meet (respectively join) semilattice $L$, any subset $X$ of $L$ has a meet (respectively a join) denoted by $\wedge X$ (respectively $\vee X)$. Also if $(L, \leq, \wedge)$ is a meet-semilattice and $\left(L^{\prime}, \leq^{\prime}, \vee^{\prime}\right)$ a join-semilattice, $L$ and $L^{\prime}$ are dual if there exists a bijection map $f$ from $L$ onto $L^{\prime}$ such that $f(x \wedge y)=f(x) \vee^{\prime} f(y)$ 
(or equivalently, if $\left[x \leq y\right.$ if and only if $\left.f(y) \leq^{\prime} f(x)\right]$ ). Such a map is called an anti-isomorphism between $L$ and $L^{\prime}$. For definitions of lattice theory not given here, see for instance [7] or [9].

We denote by $(A, \leq)$ the poset of all anti-exchange closure operators on $S$, where $A$ denotes the set of all anti-exchange closure operators on $S$ and $\leq$ the pointwise order between operators: $k \leq k^{\prime}$ if and only if $k(A) \subseteq k^{\prime}(A)$ for every $A \subseteq S$. The following lemma appears in [10] (Theorem 2.2). We give a short proof for completeness.

Lemma 1 The poset $(A, \leq)$ of all anti-exchange closure operators is a meetsemilattice where the meet of two anti-exchange closure operators $k$ and $k^{\prime}$ is their intersection $k \cap k^{\prime}$ (with $\left.\left(k \cap k^{\prime}\right)(A)=k(A) \cap k^{\prime}(A), \forall A \subseteq S\right)$.

Proof Let $k, k^{\prime} \in A$. It is easy to check that $k \cap k^{\prime}$ is an extensive and isotone operator. Since $k(A) \cap k^{\prime}(A) \subseteq k\left(k(A) \cap k^{\prime}(A)\right) \cap k^{\prime}\left(k(A) \cap k^{\prime}(A)\right) \subseteq$ $k^{2}(A) \cap k^{\prime 2}(A)=k(A) \cap k^{\prime}(A)$, then $\left(k \cap k^{\prime}\right)^{2}(A)=\left(k \cap k^{\prime}\right)(A)$ and so $k \cap k^{\prime}$ is a closure operator.

Now if $A \subseteq S$, we have $x, y \notin k(A) \cap k^{\prime}(A)$ and $y \in k(A+x) \cap k^{\prime}(A+x)$. Since $x \notin k(A)$ or $x \notin k^{\prime}(A)$, then $x \notin k(A+y)$ or $x \notin k^{\prime}(A+y)$, and so $x \notin k(A+y) \cap k^{\prime}(A+y)$. Finally $k \cap k^{\prime}$ is an anti-exchange closure operator.

We denote by $(\mathbb{P}, \leq)$ the poset of all path independent choice operators, where $\mathbb{P}$ denotes the set of all path independent choice operators and $\leq$ the pointwise order between operators.

Lemma 2 The poset $(\mathbb{P}, \leq)$ of all path independent choice operator is a join-semilattice, where the join operation of two choice operators $c$ and $c^{\prime}$ is their union $c \cup c^{\prime}$ (with $\left.\left(c \cup c^{\prime}\right)(A)=c(A) \cup c^{\prime}(A), \forall A \subseteq S\right)$.

Proof Let $c, c^{\prime} \in \mathbb{P}$. It is obvious that $c \cup c^{\prime}$ is a contracting operator. For $A, B \subseteq S$ and since $c$ is path independent (and so idempotent), one gets $c\left(\left(c \cup c^{\prime}\right)(A) \cup\left(c \cup c^{\prime}\right)(B)\right)=c\left(c(A) \cup c^{\prime}(A) \cup c(B) \cup c^{\prime}(B)\right)=c\left(c^{2}(A) \cup c c^{\prime}(A) \cup\right.$ $\left.c^{2}(B) \cup c c^{\prime}(B)\right)=c\left(c(A) \cup c c^{\prime}(A) \cup c(B) \cup c c^{\prime}(B)\right)=c\left(A \cup c^{\prime}(A) \cup B \cup c^{\prime}(B)\right)=$ $c(A \cup B)$. In the same way, $c^{\prime}(A \cup B)=c^{\prime}\left(\left(c \cup c^{\prime}\right)(A) \cup\left(c \cup c^{\prime}\right)(B)\right)$. So $\left(c \cup c^{\prime}\right)(A \cup B)=\left(c \cup c^{\prime}\right)\left(\left(c \cup c^{\prime}\right)(A) \cup\left(c \cup c^{\prime}\right)(B)\right)$, and $c \cup c^{\prime}$ is a path independent choice operator.

We now show that the meet-semilattice of anti-exchange closure operators and the join-semilattice of path independent choice operators are dual 
semilattices.

Theorem 3 Let $S$ be a set. The two maps $\epsilon\left(\epsilon_{k}(A)=\{x \in A: x \notin\right.$ $k(A-x)\})$ and $\kappa\left(\kappa_{c}(A)=\cup\{X \subseteq S: c(X)=c(A)\}\right)$ define two inverse anti-isomorphisms between the meet semilattice $A$ of anti-exchange closure operators and the join-semilattice $\mathbb{P}$ of path independent choice operators.

Proof In order to prove the theorem it is sufficient to prove the following properties:

- if the closure operator $k$ is anti-exchange (respectively if the choice operator $c$ is path independent) then $\epsilon_{k}$ is a path independent choice operator (respectively $\kappa_{c}$ is an anti-exchange closure operator);

- the compositions $\kappa \epsilon$ and $\epsilon \kappa$ of the two maps $\kappa$ and $\epsilon$ are the identity maps on $\mathbb{P}$ and on $A$;

- $\epsilon_{k \wedge k^{\prime}}=\epsilon_{k} \cup \epsilon_{k^{\prime}}, \forall k, k^{\prime} \in A$.

1) Let $k$ be an anti-exchange closure operator. It is obvious that $\epsilon_{k}$ is a contracting operator satisfying $\epsilon_{k}(A) \neq \emptyset$, for every $A \neq \emptyset$ (if $\epsilon_{k}(A)=\emptyset$, $k \epsilon_{k}(A)=k(\emptyset)=\emptyset \neq k(A)$, a contradiction with $\left.k \epsilon_{k}=k\right)$.

We show that $\epsilon_{k}$ is path independent or equivalently (Proposition $1(3 \mathrm{~d})$ ) satisfies the heritage and outcast properties. We already know (Proposition $5)$ that $\epsilon_{k}$ satisfies the heritage property. We prove that it satisfies the outcast property, i.e that $\epsilon_{k}(B) \subseteq A \subseteq B$ implies $\epsilon_{k}(B)=\epsilon_{k}(A)$. Since $k$ is an anti-exchange closure operator, the above inclusions give $k \epsilon_{k}(B)=k(B) \subseteq$ $k(A) \subseteq k(B)$ (Theorem $2(2)$ ). So $k(A)=k(B), \epsilon_{k} k(A)=\epsilon_{k} k(B)$, and $\epsilon_{k}(A)=\epsilon_{k}(B)($ Theorem $2(4))$.

2) We now show that if $c$ is a path independent choice operator then the composition of $\epsilon$ and $\kappa$ is the identity, i.e. $\epsilon_{\kappa_{c}}=c$ for every $c$ in $\mathbb{P}$.

Let $A \subseteq S$. Obviously $x \in c(A)$ if and only if $(A-x) \nsupseteq c(A)$ and if and only if $c(A-x) \neq c(A)$ (for the last sufficient condition, since $c$ satisfies the outcast property, $c(A) \subseteq A-x \subseteq A$ implies $c(A)=c(A-x))$. This is also equivalent to $\kappa_{c}(A-x) \subset \kappa_{c}(A)$ (for the necessary condition, $\kappa_{c}(A-x)=\kappa_{c}(A)$ implies $c \kappa_{c}(A-x)=c \kappa_{c}(A)$, and since $c$ is path independent, then $c(A-x)=c(A)$ by Theorem 1). Finally, this is equivalent to $x \in \epsilon_{\kappa_{c}}(A)$ (by the definition of $\epsilon_{\kappa_{c}}$ ). 
3) We prove that if $c$ is a path independent choice operator then $\kappa_{c}$ is an anti-exchange closure operator. We know that $\kappa_{c}$ is a closure operator satisfying $\kappa_{c} c=\kappa_{c}$ (Proposition 3). Now by (2) above, $c=\epsilon_{\kappa_{c}}$. So $\kappa_{c} \epsilon_{\kappa_{c}}=\kappa_{c}$ and $\kappa_{c}$ is an anti-exchange closure operator (Theorem $2(2)$ ).

4) We show that if $k$ is an anti-exchange closure operator then the composition of $\kappa$ and $\epsilon$ is the identity, i.e. $k=\kappa_{\epsilon_{k}}$ for every $k$ in $A$.

Let $A \subseteq S$. By Theorem $2(7), \kappa_{\epsilon_{k}}(A)=\bigcup\left\{X: \epsilon_{k}(X)=\epsilon_{k}(A)\right\}=$ $\bigcup\left\{X: \epsilon_{k}(A) \subseteq X \subseteq k(A)\right\}=k(A)$.

5) Finally, we prove that if $k, k^{\prime}$ are anti-exchange closure operators then $\epsilon_{k \wedge k^{\prime}}=\epsilon_{k} \cup \epsilon_{k^{\prime}}$. Let $A \subseteq S$. We have $\left(k \wedge k^{\prime}\right)(A)=k(A) \cap k^{\prime}(A)$.

$x \in \epsilon_{k \wedge k^{\prime}}(A)$ if and only if $x \notin\left(k \wedge k^{\prime}\right)(A-x)=k(A-x) \cap k^{\prime}(A-x)$ if and only if $x \notin k(A-x)$ or $x \notin k^{\prime}(A-x)$ if and only if $x \in \epsilon_{k}(A) \cup \epsilon_{k^{\prime}}(A)$.

In the proof of the above theorem, we have shown that if $c$ is a path independent choice operator, then $\kappa_{c}$ is an anti-exchange closure operator. The converse implication is false, as it is shown in the example of Remark 2. We have also shown that if $k$ is an anti-exchange closure operator, then $\epsilon_{k}$ is a path independent choice operator. Here there is a converse implication which gives other characterizations of such a closure operator.

Proposition 6 Let $k$ be a closure operator such that $k(\emptyset)=\emptyset$. The following properties are equivalent :

(1) $k$ is an anti-exchange closure operator,

(2) $\epsilon_{k}$ is a path independent choice operator : $\epsilon_{k}(A \cup B)=\epsilon_{k}\left(\epsilon_{k}(A) \cup \epsilon_{k}(B)\right)$, $\forall A, B \subseteq S$.

(3) $\epsilon_{k}$ satisfies the following property : $\epsilon_{k}(A) \subseteq B \subseteq A$ implies $\epsilon_{k}(B) \subseteq$ $\epsilon_{k}(A)$, for all $A, B \subseteq S$.

Proof $(1) \Rightarrow(2)$ : See theorem (3)

$(2) \Rightarrow(3)$ : Obvious (since by Proposition 1 (3d), the path independence property implies the stronger outcast property).

$(3) \Rightarrow(1)$ : We already know that $\epsilon_{k}$ satisfies the heritage property (Proposition 5). Then if $\epsilon_{k}(A) \subseteq B \subseteq A, \epsilon_{k}(A) \cap B=\epsilon_{k}(A) \subseteq \epsilon_{k}(B)$. Hence, by hypothesis, $\epsilon_{k}(A)=\epsilon_{k}(B)$ and $\epsilon_{k}$ satisfies the outcast property. Now assume that $k$ is not anti-exchange. There exists a closed set $K$ of $k$ such that $x, y \notin K, y \in k(K+x)$ and $x \in k(K+y)$. Then $y \notin \epsilon_{k}(K+\{x, y\})$, and $x \notin \epsilon_{k}(K+\{x, y\})$. So $\epsilon_{k}(K+\{x, y\}) \subseteq K \subset K+x \subset K+\{x, y\}$, and by 
the outcast property, $\epsilon_{k}(K+x)=\epsilon_{k}(K+\{x, y\})$. But since $x \notin K$, we have $x \in \epsilon_{k}(K+x)=\epsilon_{k}(K+\{x, y\})$ which is a contradiction.

Remark 6 The implication $(1) \Rightarrow(2)$ of the above proposition is the one given by Theorem 4 in [18].

We now introduce a significant subset of the set $A$ of all anti-exchange closure operators and we identify the corresponding path independent choice operators as the choice operators satisfying the concordance property defined below.

Definition $8 \mathrm{~A}$ closure operator $k$ on $S$ is called a $T_{0}$-closure operator if it satisfies the two following properties :

(a) $\forall x, y \in S, k(x)=k(y) \Rightarrow x=y$,

(b) $\forall A, B \subseteq S, k(A \cup B)=k(A) \cup k(B)$.

This terminology is justifed by the fact that the family $\mathcal{F}_{k}$ of the closed sets of a $T_{0}$-closure operator is a $T_{0}$-topology, i.e. a family of subsets of $S$ closed by union and intersection, containing $\emptyset$ and $S$, and satisfying the above property (a). So $\left(\mathcal{F}_{k}, \subseteq\right)$ is a distributive lattice.

Definition 9 Let $c$ be a choice operator on $S$. c satisfies the concordance property (C) if for all $A, B \subseteq S, c(A) \cap c(B) \subseteq c(A \cup B)$.

Remark 7 In the theory of choice functions, the concordance property has also been called the expansion property or the property $\gamma$.

Proposition 7 The following holds:

(1) A $T_{0}$-closure operator is an anti-exchange closure operator,

(2) Let $k$ be a closure operator. $k$ is a $T_{0}$-closure operator if and only if $\epsilon_{k}$ is a path independent choice operator satisfying the concordance property.

Proof (1) Let $k$ be a $T_{0}$-closure operator on $S$ and $A \subseteq S$. Assume that there exists $x, y$ with $x \neq y, x, y \notin k(A), x \in k(A+y)=k(A) \cup k(y)$ and $y \in k(A+x)=k(A) \cup k(x)$. Then, $x \in k(y), y \in k(x)$, so $k(x)=k(y)$ and $x=y$, which is a contradiction.

(2) Let $k$ be a $T_{0}$-closure operator on $S, A, B \subseteq S$ and $x \in \epsilon_{k}(A) \cap \epsilon_{k}(B)$. So $k(A-x) \subset k(A), k(B-x) \subset k(B)$ and $k(A \cup B-x)=k(A-x) \cup k(B-x) \subset$ $k(A) \cup k(B)=k(A \cup B)$, i.e. $x \in \epsilon_{k}(A \cup B)$.

Conversely, assume that $\epsilon_{k}$ is a path independent choice operator on $S$ 
satisfying the concordance property. By Proposition $6, k$ is an anti-exchange closure operator and so it satisfies condition (a) of the definition of a $T_{0^{-}}$ closure operator (indeed it is easy to check that any anti-exchange closure operator satisfies this condition).

In order to prove that $k$ satisfies condition (b) of Definition 8 , we first show that for every $A \subseteq S, k(A)=\bigcup\left\{k(x), x \in \epsilon_{k}(A)\right\}$. If not, take any $z \in k(A)-\bigcup\left\{k(x), x \in \epsilon_{k}(A)\right\}$. If for $x \in \epsilon_{k}(A), z \notin \epsilon_{k}(z \cup x)$, then $z \in k(x)=k(z \cup x)$, which is a contradiction. So $z \in \bigcap\left\{\epsilon_{k}(z \cup x)\right.$, $\left.x \in \epsilon_{k}(A)\right\}$ and by the concordance property, $z \in \epsilon_{k}\left(z \cup \epsilon_{k}(A)\right)$. Then $k\left(\epsilon_{k}(A)\right)=k(A) \subset k\left(z \cup \epsilon_{k}(A)\right)=k(A)$, another contradiction. We now show that $k(A \cup B)=k(A) \cup k(B)$. Indeed by the property shown just above $k(A \cup B)=\bigcup\left\{k(x), x \in \epsilon_{k}(A \cup B)\right\}=\bigcup\left\{k(x), x \in \epsilon_{k}\left(\epsilon_{k}(A) \cup \epsilon_{k}(B)\right)\right\} \subseteq$ $\bigcup\left\{k(x), x \in \epsilon_{k}(A) \cup \epsilon_{k}(B)\right\}=k(A) \cup k(B) \subseteq k(A \cup B)$ (by the properties of a closure operator), so $k(A \cup B)=k(A) \cup k(B)$.

Remark 8 a) Point (1) of the previous proposition is well known. Point (2) is another formulation of some Koshevoy results and follows his proofs (see Proposition 3,4 and Corollary 2) in [18]. Koshevoy also gives several other characterizations of a $T_{0^{-}}$closure operator $k$ based on properties of $\epsilon_{k}$.

b) The poset of all $T_{0}$-closure operators on $S$ (endowed with the pointwise order) is also a meet-semilattice, but it is not a sub-meet-semilattice of $(A, \leq)$. The dual poset of choice operators satisfying the path independence and concordance properties is a join-semilattice, but not a sub-joinsemilattice of $(\mathbb{P}, \leq)$. This second semilattice is isomorphic with the $U$ semilattice of $T_{0}$-topologies on $S$, while, according to a well-known Birkhoff result $([6])$, the first one is isomorphic with the $\bigcap$-semilattice of partial orders on $S$.

\section{The ordinal representations of the anti-exchange closure operators and of the path independent choice operators}

In section 2, anti-exchange closure operators have been defined axiomatically. Here we first recall the theory of representation of these operators by "concrete" closure operators associated with partial orders. Then we will translate this theory to path independent choice operators by using the duality obtained in section 3 . 
Let $\leq$ be a partial order on $S$, and $A \subseteq S$. We denote by $\operatorname{Max}\left(\leq_{/ A}\right)$ the set $\{x \in A: \nexists y \in A$ with $x<y\}$ of the maximal elements of $A$ with respect to the partial order $\leq$. It is clear that the operator $k_{\leq}$defined on $S$ by $k_{\leq}(A)=\{y \in S: \exists x \in A$ with $y \leq x\}$ is a closure operator (the closed sets of $k_{\leq}$are the so-called order ideals or down sets of $\left.\leq\right)$. It is also clear that an element of $A$ is extreme, with respect to this closure operator $k_{\leq}$, if and only if it is a maximal element of $A: \epsilon_{k_{\leq}}(A)=\operatorname{Max}\left(\leq_{/ A}\right)$. In the particular case where $\leq$ is a linear order, $\operatorname{Max}\left(\leq_{/ A}\right)$ contains only one element equal to the maximum element of $A$.

Definition 10 An order (respectively linear) closure operator $k$ on $S$ is a closure operator on $S$ such that there exists a partial order (respectively a linear order) $\leq$ on $S$ with $k=k_{\leq}$.

It is obvious that an order closure operator is an anti-exchange closure operator. Especially, in the semilattice $A$ of anti-exchange closure operators, the meet of linear closure operators is an anti-exchange closure operator. The converse is also true: given any anti-exchange closure operator $k$, there exists a set of linear orders on $S$ such that $k$ is the meet of the associated linear closure operators. Moreover, the minimum number of linear orders required in such a representation of $k$ is known (see [14]). This minimum number can be simply expressed by considering the family $\mathcal{F}_{k}$ of the closed sets of the closure operator $k$. We say that a closed set $K$ of $k$ is $\bigcap$-irreducible (with respect to $k$ ), if it cannot be obtained as the intersection of other closed sets of $k$ (i.e. if $K=K_{1} \cap K_{2}$ with $K_{1}, K_{2} \in \mathcal{F}_{k}$ implies $K=K_{1}$ or $\left.K=K_{2}\right)$. We note $\left(\mathcal{M}_{k}, \subseteq\right)$ the poset of all $\bigcap$-irreducible closed sets of $k$. For any poset $(P, \leq)$, the maximum number of incomparable elements of this poset is called the width of $(P, \leq)$.

We can now state the significant results of the theory of the representation of anti-exchange closure operators by linear closure operators.

Theorem 4 (1) In the meet-semilattice $A$ of anti-exchange closure operators, any anti-exchange closure operator is a meet of linear closure operators $: k \in A$ implies that there exists a set $\mathcal{L}=\left\{\leq_{1}, \ldots, \leq_{i}, \ldots, \leq_{p}\right\}$ of linear orders on $S$ such that $k=\bigwedge_{\leq_{i} \in \mathcal{L}}\left(k_{\leq_{i}}\right)$.

(2) The minimum number of linear orders required in such a representation of the anti-exchange closure operator $k$ is the width of the poset $\left(\mathcal{M}_{k}, \subseteq\right)$ of the $\cap$-irreducible closed sets of $k$. 
(3) Especially if $k=k_{\leq}$is the order closure operator associated with the partial order $\leq$, the minimum number of linear orders required in such a representation of $k$ is the width of the poset $(S, \leq)$.

Proof For (1) and (2) see Theorem 4.1 in [14] and also [23],(p.96). (3) is a consequence of (2) since the lattice of closed sets of $k_{\leq}$is the lattice of order ideals of $\leq$, and since it is well known $([7],[9])$ that the poset $\left(M_{k_{\leq}}, \subseteq\right)$ of the meet-irreducible elements of this lattice is isomorphic with the poset $(S, \leq)$.

We can now use the duality described in section 3 to translate these results into results on the representation of path independent choice operators. We need the following definition and lemma.

Definition 11 An order (respectively linear) choice operator c on $S$ is a choice operator such that there exists a partial order (respectively a linear order $) \leq$ on $S$ with $c(A)=\operatorname{Max}\left(\leq_{/ A}\right)$ for every $A \subseteq S$. Such an operator is denoted by $c_{\leq}$.

Lemma 3 In the duality between the anti-exchange closure operators and the path independent choice operators, the order closure operators correspond with the order choice operators.

Proof If $k_{\leq}$is the closure operator associated with the partial order $\leq$, $\epsilon_{k_{\leq}}=c_{\leq}\left(\right.$since $\left.\epsilon_{k_{\leq}}(A)=\operatorname{Max}(\leq / A)=c_{\leq}(A)\right)$. Conversely if $c_{\leq}$is the choice operator associated with the partial order $\leq$, then $\kappa_{c_{\leq}}=k_{\leq}$(since $\kappa_{c_{\leq}}(A)=\{X \subseteq S: \operatorname{Max}(\leq / X)=\operatorname{Max}(\leq / A)\}=\{y \in S: \exists x \in A$ with $\left.y \leq x\}=k_{\leq}(A)\right)$.

The following corollary of Theorem 4 gives immediately the representation theorem for path independent choice operators obtained by Aizerman and Malishevski ([3]) and allows us to find the minimum cardinality of these representations.

Corollary 1 (1) In the semilattice IP of path independent choice operators, any path independent choice operator is a join of linear choice operators :

$c \in \mathbb{P}$ implies that there exists a set $\mathcal{L}=\left\{\leq_{1}, \ldots, \leq_{i}, \ldots, \leq_{p}\right\}$ of linear orders on $S$ such that for every $A \subseteq S, c(A)=\bigcup_{\leq_{i} \in \mathcal{L}} \operatorname{Max}\left(\left(\leq_{i}\right)_{/ A}\right)$,

(2) The minimum number of linear orders required in such a representation of the path independent choice operator $c$ is the width of the poset $\left(\mathcal{M}_{\kappa_{c}}, \subseteq\right)$ of 
the $\bigcap$-irreducible closed sets of the associated anti-exchange closure operator $\kappa_{c}$.

(3) Especially if $c$ is an order choice operator associated with the partial order $\leq, c$ is a join of linear choice operators and the minimum number of linear orders required in such a representation of $c$ is the width of the poset $(S, \leq)$.

Proof (1) Let $c$ be a path independent choice operator and $\kappa_{c}$ its associated anti-exchange closure operator. By Theorem $4(1)$, there exists a set $\mathcal{L}=\left\{\leq_{1}, \ldots, \leq_{i}, \ldots, \leq_{p}\right\}$ of linear orders on $S$ such that $\kappa_{c}=\bigwedge_{\leq_{i} \in \mathcal{L}}\left(k_{\leq_{i}}\right)$. By the properties of the duality between the semilattices $A$ and $\mathbb{P}, c=\epsilon_{\kappa_{c}}=$ ${ }^{\epsilon} \bigwedge_{\leq_{i} \in \mathcal{L}}\left(k_{\leq_{i}}\right)=\bigcup_{\leq_{i} \in \mathcal{L}}\left(\left(\epsilon_{\left.k_{\leq_{i}}\right)=\bigcup_{\leq_{i} \in \mathcal{L}}\left(c_{\leq_{i}}\right)}\right.\right.$, i.e. $c(\bar{A})=\bigcup_{\leq_{i} \in \mathcal{L}} \operatorname{Max}\left(\left(\leq_{i}\right)_{/ A}\right)$.

(2) By the same duality, if $c=\bigcup_{\leq_{i} \in \mathcal{L}}\left(c_{\leq_{i}}\right)$, then $\kappa_{c}=\bigwedge_{\leq_{i} \in \mathcal{L}}\left(k_{\leq_{i}}\right)$, and so the result follows immediately from Theorem 4 (2).

(3) Obvious by Theorem 4 (3) and Lemma 3.

The fact that any path independent closure operator can be obtained as a union of linear choice operators first appeared in an Aizerman and Malishevski paper ([3], see also [2]). Given an anti-exchange closure operator $c$, the usual proof of this result is to construct the set $\mathcal{L}$ of all linear orders $L$ such that $c_{L} \leq c$, and to prove that $c=\bigvee\left\{c_{L}, L \in \mathcal{L}\right\}$. This set $\mathcal{L}$ is in a one-to-one correspondence with the set of all maximal chains of the convex geometry $\mathcal{F}_{\kappa_{c}}$ associated with the anti-exchange closure operator $\kappa_{c}$. Using the duality, one has more generally $c=\bigvee\left\{c_{L}, L \in \mathcal{L}^{\prime} \subseteq \mathcal{L}\right\}$ if and only if the maximal chains of $\mathcal{F}_{\kappa_{c}}$ corresponding with the linear orders of $\mathcal{L}^{\prime}$ contain all the $\bigcap$-irreducible closed sets of $\kappa_{c}$.

The problem to find the minimum size of such a representation was investigated by Aleskerov, Zavalishin and Litvakov ([4]) for any order choice operator and by Litvakov ([20]) for an arbitrary path independent choice operator (see also [1]). In the case of an order choice operator $c$ associated with the order $\leq$, the authors of [4] get the result obtained by our duality approach: the minimum size of a representation of $c$ by linear orders is the width of the poset $(S, \leq)$. In the case of a path independent choice operator $c$ defined on $S$, Litvakov defines a partial order on a new set containing "before replicas" of the elements of $S$ and he shows that $c$ coincides with the order choice operator associated with this partial order. Then applying the result of [4] he gets that the minimum size of a representation of $c$ by linear orders is the width of this poset. Since it is not obvious that this result is 
equivalent to the result brought by point 2 of the above corollary, we are going to describe the Litvakov result and show the equivalence of the two results. This will give also another way to construct the convex geometry (and so the anti-exchange closure operator) associated with a path independent choice operator.

Let $c$ be a choice operator on $S$ and $x \in S$. We say that a subset $D$ of $S$ is an $x$-dominating set (with respect to $c$ ) if $S-D$ is a maximal subset of $S$ such that $x$ is chosen in this subset : $x \in c(S-D), x \notin c(X)$ if $X \supset S-D$. We set $\mathcal{D}(x)=\{x$-dominating sets $\}$. An $x$-dominating set $D$ will be also denoted by $D_{x}$. Remark that $\mathcal{D}(x)$ is a Sperner family $\left(D_{x}, D_{x}^{\prime} \in \mathcal{D}(x)\right.$ imply $D_{x} \nsubseteq D_{x}^{\prime}$ and $\left.D_{x}^{\prime} \nsubseteq D_{x}\right)$. Remark also that $\mathcal{D}(x)=\mathcal{D}(y)$ does not imply $x=y$, since, for instance, $x$ and $y \in c(S)$ imply $\mathcal{D}(x)=\mathcal{D}(y)=\{\emptyset\}$. We set $\mathcal{D}^{\times}(x)=\left\{\left(x, D_{x}\right), D_{x} \in \mathcal{D}(x)\right\}$ and $\mathcal{D}^{\times}=\bigcup\left\{\mathcal{D}^{\times}(x), x \in S\right\}$ (this definition, lightly different from the Litvakov definition, allows to not consider replicas). Finally we define a relation $<$ on $\mathcal{D}^{\times}$by $:\left(x, D_{x}\right)<\left(y, D_{y}\right)$ if $\{x\} \cup D_{x} \subseteq D_{y}$. It is easy to check that $<$ is an order relation on $\mathcal{D}^{\times}$. The Litvakov result says that if $c$ is a path independent choice operator, the minimum number of linear orders required to obtain $c$ (as a union of linear choice operators) is the width of the poset $(\mathcal{D},<)$. Recall that two posets $(P,<)$ and $\left(P^{\prime},<^{\prime}\right)$ are dual if there exists a one-to-one map $f$ from $P$ onto $P^{\prime}$ such that $x<y$ if and only if $f(y)<^{\prime} f(x)$. Since two dual posets have the same width, the equivalence between this result and point 2 of corollary 1 comes from the following proposition.

Proposition 8 Let $c$ be a path independent choice operator, and $\kappa_{c}$ its associated anti-exchange closure operator. The poset $\left(\mathcal{D}^{\times}(x),<\right)$ of all the ordered pairs $\left(x, D_{x}\right)$ with $D_{x} x$-dominating set and $x \in S$, and the poset $\left(\mathcal{M}_{\kappa_{c}}, \subset\right)$ of the $\bigcap$-irreducible closed sets of $\kappa_{c}$ are dual. lemma.

In order to prove this proposition we need the following definitions and

Definition 12 Let $k$ be a closure operator on $S, \mathcal{F}_{k}$ the family of its closed sets and $x \in S$. We say that a closed set $C$ is an $x$-copoint (with respect to $k$ ) if $C$ is a maximal closed set such that $x$ does not belong to this closed set $\left(x \notin C\right.$ and $x \in k(K)$ if $K \in \mathcal{F}_{k}$ and $\left.K \supset C\right)$. We denote by $\mathcal{C}(x)$ the (Sperner) family of the $x$-copoints.

It is well known (and easy to prove) that a closed set $K$ of $k$ is an $x$ copoint for some $x$ of $S$ if and only if $K$ is an $\bigcap$-irreducible closed set of $k$. So $\bigcup\{\mathcal{C}(x), x \in S\}=\mathcal{M}_{k}$. 
Lemma 4 Let $k$ be an anti-exchange closure operator on $S$ and $\epsilon_{k}$ the associated path independent choice operator (associating with any set $A$ its set of extreme elements). The map $D \longmapsto S-(D+x)$ is a one-to-one correspondence between the family $\mathcal{D}(x)$ of the $x$-dominating sets (with respect to $\epsilon_{k}$ ) and the family $\mathcal{C}(x)$ of the $x$-copoints (with respect to $k$ ).

Proof : Recall that if $K$ is a closed set of $k, x \in \epsilon_{k}(K)$ if and only if $K-x$ is a closed set of $k$.

We first show that if $D_{x}$ is an $x$-dominating set (with respect to $\epsilon_{k}$ ), $C=$ $S-\left(D_{x}+x\right)$ is an $x$-copoint (with respect to $k$ ). First $C^{+}=C+x$ is a closed set of $k$. Indeed if $k\left(C^{+}\right) \supset C^{+}=S-D_{x}$, then $x \in \epsilon_{k}\left(C^{+}\right)=\epsilon_{k}\left(k\left(C^{+}\right)\right)$ (Theorem 2 (4)), a contradiction with the fact that $D_{x}$ is an $x$-dominating set. So since $C^{+}$is a closed set and $x \in \epsilon_{k}\left(C^{+}\right)$, then $C=C^{+}-x$ is a closed set of $k$. Assume that $C$ is not an $x$-copoint. Then, by definition of an $x$-copoint, there exists an $x$-copoint $C^{\prime}$ strictly containing $C$. So $C^{\prime}$ is an $\bigcap$-irreducible closed set of $k, C^{\prime}+x$ is the unique closed set covering $C^{\prime}$ and $x \in \epsilon_{k}\left(C^{\prime}+x\right)$. But $C^{\prime}+x \supset C^{+}=S-D_{x}$, a contradiction with $D_{x}$ $x$-dominating set.

Now we show that if $C$ is an $x$-copoint (with respect to $k$ ), $D_{x}=S-(C+x)$ is an $x$-dominating set (with respect to $\epsilon_{k}$ ). Since $C$ is an $\bigcap$-irreducible closed set of $k$, it is covered in the lattice $\mathcal{F}_{k}$ by a unique closed set denoted by $C^{+}$. Since $k$ is an anti-exchange closure operator, then $C^{+}=C+x$ (in such a lattice, $K$ and $K^{\prime}$ closed sets and $K$ covered by $K^{\prime}$ implies $\left|K^{\prime}-K\right|=1$ ), and so $x \in \epsilon_{k}\left(C^{+}\right)$, i.e. $x \in \epsilon_{k}\left(S-D_{x}\right)$. Let $X \supset S-D_{x}\left(=C^{+}\right)$and $K=k(X)$ the closure of $X$. Since $k$ is an anti-exchange closure operator, $\epsilon_{k}(X)=\epsilon_{k}(k(X))$ (Theorem $\left.2(4)\right)$. So if $x \in \epsilon_{k}(X)$, we have $x \in \epsilon_{k}(k(X))$ and $k(X)-x$ is a closed set containing $C$, which is a contradiction with the definition of an $x$-copoint $C$. So $X \supset S-D_{x}$ implies $x \notin \epsilon_{k}(X)$ and $D_{x}$ is an $x$-dominating set. From these two results one immediately gets that the map $D \longmapsto S-(D+x)$ is a one-to-one correspondence between the family $\mathcal{D}(x)$ of the $x$-dominating sets and the family $\mathcal{C}(x)$ of the $x$-copoints.

Proof of Proposition 8 : Let $c$ be a path independent choice operator and $\kappa_{c}$ its associated anti-exchange closure operator. First we define a map $\mu$ between the poset $\left(\mathcal{D}^{+},<\right)$of all the ordered pairs $\left(x, D_{x}\right)$ with $D_{x} \in \mathcal{D}(x)$ and $x \in S$, and the poset $\left(\mathcal{M}_{\kappa_{c}}, \subset\right)$ of the $\bigcap$-irreducible closed sets of $\kappa_{c}$. For $\left(x, D_{x}\right) \in \mathcal{D}^{+}, \mu\left[\left(x, D_{x}\right)\right]=S-\left(D_{x}+x\right)$. By Lemma $4, S-\left(D_{x}+x\right) \in \mathcal{M}_{\kappa_{c}}$ (indeed it is an $x$-copoint of $\kappa_{c}$ ) and the map $\mu$ is a one-to-one correspondence between $\mathcal{D}^{+}$and $\mathcal{M}_{\kappa_{c}}$. Let us now consider $\left(x, D_{x}\right)$ and $\left(x, D_{y}\right) \in \mathcal{D}^{+}$, with 
$\left(x, D_{x}\right)<\left(y, D_{y}\right)$, i.e. $x \cup D_{x} \subseteq D_{y}$. So $S-\left(D_{x}+x\right) \supseteq S-D_{y} \supset S-\left(D_{y}+y\right)$, i.e. $\mu\left[\left(x, D_{x}\right)\right] \supset \mu\left[\left(y, D_{y}\right)\right]$. Conversely let $C_{x}=\mu\left[\left(x, D_{x}\right)\right]=S-\left(D_{x}+x\right)$ be an $x$-copoint and $C_{y}=\mu\left[\left(x, D_{x}\right)\right]=S-\left(D_{y}+x\right)$ an $y$-copoint. Assume that $C_{x} \supset C_{y}$. Then $y \in C_{x}, y \notin C_{y}$ and so this inclusion is equivalent to $C_{x} \supseteq C_{y}+y$, and also equivalent to $S-C_{x}=\{x\} \cup D_{x} \subseteq S-\left(C_{y}+y\right)=D_{y}$ i.e. equivalent to $\left(x, D_{x}\right)<\left(y, D_{y}\right)$. Then we have shown that the map $\mu$ is an anti-isomorphism between the posets $\left(\mathcal{D}^{+},<\right)$and $\left(\mathcal{M}_{\kappa_{c}}, \subset\right)$.

Remark 9 A well-known Birkhoff result ([6]) says that a closure operator is an order closure operator if and only if it is a $T_{0}$-closure operator. Then using the duality between the order closure operators and the order choice operators (Lemma 3) and Proposition 7, one gets another classical result of the theory of choice functions, due to Schwartz ([27]) :

a choice operator $c$ satisfies the heritage, outcast and concordance properties if and only if there exists a partial order $\leq$ on $S$ such that $c(A)=\operatorname{Max}\left(\leq_{/ A}\right)$, for every $A \subseteq S$.

\section{Conclusion}

We have shown in Section 3 of this paper that the correspondence between anti-exchange closure operators and path independent choice operators dicovered by Koshevoy and Johnson and Dean is a duality between two semilattices, which implies that any result in the theory of anti-exchange closure operators can be automatically translated into a result in the theory of path independence choice operators (and conversely). In doing this translation for some results of the first theory, we have obtained results of the theory of choice operators (section 4). Let us recall some related lines of development of this last theory, called the theory of choice functions in microeconomics. It first dealt with the so-called rationalizable choice functions, i.e. the choice functions $c$ such that there exists an (acyclic) binary (preference) relation $R$ on the set $S$ of given outcomes satisfying $c(A)=\operatorname{Max}\left(R_{/ A}\right)$ for all $A$ contained in $S$. In 1971 Sen ([28]) showed that a choice function is rationalizable if and only if it satisfies the heritage and concordance properties. A rationalizable choice function such that the relation $R$ is a partial order has been called Pareto (or quasi-transitively) rationalizable. Indeed any partial order $\leq$ can be obtained as the intersection of a set $\mathcal{L}$ of linear orders, and $\operatorname{Max}\left(\leq_{/ A}\right)$ is the set of the Pareto optimal elements with respect to $\mathcal{L}$. The problem to find the minimum number of linear orders required in such a 
representation of a Pareto rationalizable choice function is then the problem to find the minimum number of linear orders whose intersection is equal to a given partial order, i.e. the famous (and generally very difficult) problem of the dimension of a partial order (see [31]). The axiomatic characterization of the Pareto rationalizable choice functions by the heritage, concordance and outcast properties (Remark 9) is due to Schwartz ([27]). Since an arbitrary path independent choice operator does not satisfy the concordance property, it is not rationalizable in the above sense. But the 1981 Aizerman and Malishevski representation theorem ([2], [3]) shows that such a path independent choice operator can be obtained by a rational mechanism called by them the joint-extremal mechanism (and by some authors the pseudorationalizable mechanism). The use of the duality between anti-exchange closure operators and path independent choice operators gives immediately this result and the minimum number of linear orders required in such a representation (Corollary 1). This last result, proved equivalent to a Litvakov result $([20])$, shows that the problem to find such a minimum representation is polynomial. Indeed there exists polynomial algorithms to find the meet irreducible elements of a lattice and the width of a poset.

An open question is to know if there exists other interesting connections between the theory of closure operators and the theory of choice operators. For instance one could define classes of choice operators by the property that their associated extensive operator $\kappa_{c}$ is a closure operator or a closure operator satisfying some properties. Are they interesting classes of choice operators ? If not, do they contain other interesting subclasses that the class of path independent choice operators ? Remark that it is easy to find examples of choice operators for which $\kappa_{c}$ is a closure operator (and even an anti-exchange closure operator) but that have none of the heritage, concordance and outcast properties. One can also raise these questions for contracting operators which are not choice operators in the sense of this paper : $c(A)$ can be empty for $A$ non-empty (these more general choice functions have been also considered in the theory of revealed preference and in this case the choice operators of this paper are sometimes called proper choice functions). We will try to investigate such questions in the future.

Acknowledgments. The authors wish to thank F. Aleskerov and an anonymous referee for their helpful suggestions and comments. 


\section{References}

[1] Aizerman M.A., Aleskerov F., (1995). In: Theory of choice. NorthHolland, Elsevier, Amsterdam.

[2] Aizerman M.A., Malishevski A.V., (1977). Structural properties in the theory of choice of variants. In: Proceedings of the $7^{\text {th }}$ All Union Seminar on Control Problems, Book 2, IPU, Minsk, 93-97 (in Russian).

[3] Aizerman M.A., Malishevski A.V., (1981). General Theory of best variants choice. IEEE Trans. Automatic Control AC-26(5), 1030-1040.

[4] Aleskerov F.T., Zavalishin N.V. and Litvakov B.M., (1979). On decomposition of choice functions on a system of simpler functions. Automation and Remote Control, 3, 409-419.

[5] Bilbao J.M., Jimenez A., Lopez J.J. (1998). The Banzhaf power index on convex geometries. Mathematical Social Sciences, 36(2), 157-173.

[6] Birkhoff G., (1937). Rings of sets. Duke Mathematical Journal, 3 , 443454.

[7] Birkhoff G., (1967). Lattice Theory (third ed.). Amer. Math.Soc., Providence, RI.

[8] Caspard N., Monjardet B., (2000). The lattice of closure systems, closure operators and implicational systems on a finite set : a survey. To appear.

[9] Davey B.A., Priestley H.A., (1990). Introduction to lattices and order. Cambridge University Press, Cambridge.

[10] Edelman P.H., (1980). Meet-distributive lattices and the anti-exchange closure. Algebra Universalis, 10, 290-299.

[11] Edelman P.H., (1986). Abstract convexity and meet-distributive lattices. Contemporary Mathematics, 57, 127-150.

[12] Edelman P.H., (1997). A note on voting. Mathematical Social Sciences, $34(1), 37-50$.

[13] Edelman P.H., Jamison R.E., (1985). The theory of convex geometries. Geometriae Dedicata 19, 247-270. 
[14] Edelman P.H., Saks M.E., (1988). Combinatorial representation and convex dimension of convex geometries. Order, 5(1), 23-32.

[15] Johnson M.R., Dean R.A., (1996). An algebraic characterization of path independent choice functions. In: Third international meeting of the Society for Social Choice and Welfare, Maastricht, The Netherlands.

[16] Johnson M.R., Dean R.A., (2000). Locally complete path independent choice functions and their lattices. To appear in Mathematical Social Sciences.

[17] Koppen M., (1988). On alternative representations for knowledge spaces. Mathematical Social Sciences, 36(2), 127-143.

[18] Korte B., Lovasz L. and Schrader R., (1991). Greedoids. Springer Verlag, Berlin.

[19] Koshevoy G. A., (1999). Choice functions and abstract convex geometries. Mathematical Social Sciences, 38(1), 35-44.

[20] Litvakov B.M., (1981). Minimal representation of joint-extremal choice of options (Plott's Functions). Automation and Remote Control, 1, 182184.

[21] Malishevski A.V., (1994). Path-independent in serial-parallel data processing. Mathematical Social Sciences, 27, 335-367.

[22] Malishevski A.V., (1996). Structural characterization of the path independence property for set transformations. In: E. Diday, Y. Lechevallier, O. Opitz, eds.), Ordinal and Symbolic Data Analysis. Studies in Classification, Data Analysis and Knowledge Organization.Springer, Berlin, pp. 319-327.

[23] Matalon B., (1965). L'analyse hiérarchique. Gauthier-Villars Paris.

[24] Monjardet B., (1990). The consequences of Dilworth's work on lattices with unique irreducible decompositions. In: Bogart K., Freese R., Kung J. (Eds.), The Dilworth theorems ; Selected works of Robert P. Dilworth. Birkhaüser, 192-201.

[25] Moulin H., (1985). Choice functions over a finite set : A summary. Social Choice and Welfare, 2(2), 147-160. 
[26] Plott C.R., (1973). Path independence, rationality and social choice. Econometrica, 41(6), 1075-1091.

[27] Schwartz T., (1976). Choice functions, "rationality" conditions and variations on the weak axiom of revealed preference. Journal of Economic Theory, 13, 414-427.

[28] Sen A.K., (1971). Choice functions and revealed preference. Review of Economic Studies, 38(3), 307-317.

[29] Soltan V., (1984). Abstract convexity. Shtiintsa, Kishinev [in Russian].

[30] Suzumura K., (1983). Rational choice, collective decisions and social welfare. Cambridge University Press, Cambrige.

[31] Trotter W.T., (1992). Combinatorics and partially ordered sets: Dimension theory. Johns Hopkins University Press, Baltimore, MD. 